\title{
A Retrospective Analysis of Dialysis Events over a 3-Year Period in an Outpatient Dialysis Unit in the State of Kuwait
}

\author{
Moustapha Ahmed Ramadan ${ }^{a, b}$ Gautam Hebbar ${ }^{a}$ \\ anfection Control Directorate, Ministry of Health, Kuwait City, Kuwait; ${ }^{b}$ Department of Community Medicine, \\ Faculty of Medicine, Alexandria University, Alexandria, Egypt
}

\section{Significance of the Study}

- In this study, patients who received hemodialysis through tunneled or nontunneled central lines had a higher rate of antimicrobial use, blood stream infections and vascular access site inflammation, than patients whose hemodialysis was through arteriovenous grafts or fistulas. Hence, patients on hemodialysis through tunneled or nontunneled central lines should be encouraged to switch to grafts or arteriovenous fistulas.

\section{Keywords}

Dialysis event · Infection control · Surveillance ·

Hemodialysis · Kuwait

\begin{abstract}
Objective: To determine the difference in the rates of dialysis events stratified by vascular access type and to describe the microbiological profile and sensitivity patterns of positive blood cultures over a 3-year period. Subjects and Methods: The dialysis event data of 10,751 chronic hemodialysis patients collected from March 2013 to February 2016 at an outpatient dialysis unit in Kuwait were reviewed. The dialysis events studied were: intravenous (IV) antimicrobial use, a positive blood culture, and signs of inflammation at the vascular access site. Dialysis event rates were stratified by the type of vascular access used for the dialysis, i.e., fistula, graft, and tunneled/nontunneled central line. Rates were ex-
\end{abstract}

pressed per 100 patient-months. Results: The overall dialysis event rate was (10.7/100 patient-months). The rate of IV antimicrobial use was higher (12.53/100 patient-months) in patients with tunneled central lines than in all other vascular access types (10.29/100 patient-months). Positive blood culture and inflammation at the vascular access site were highest in patients with nontunneled central lines (1.65 and $1.54 / 100$ patient-months, respectively) when compared to those with other types of vascular access. Gram-negative rod isolates were predominant in patients with central lines $(n=$ $35 ; 46.67 \%$ ); however, common skin commensals and gramnegative rods were also identified in patients with fistula or graft ( $n=4 ; 44.45 \%)$. Conclusion: Dialysis event rates were higher among patients with tunneled or nontunneled central lines than in patients with fistula or graft. Gram-negative rods were the most commonly isolated microbial group.

(c) 2018 The Author(s)

Published by S. Karger AG, Basel

\begin{tabular}{ll}
\hline KARGER & $\begin{array}{l}\text { () } 2018 \text { The Author(s) } \\
\text { Published by S. Karger AG, Basel }\end{array}$ Openger \\
E-Mail karger@karger.com & $\begin{array}{l}\text { This is an Open Access article licensed under the Creative Commons } \\
\text { Attribution-NonCommercial-4.0 International License (CC BY-NC) } \\
\text { (http://www.karger.com/Services/OpenAccessLicense), applicable to } \\
\text { the online version of the article only. Usage and distribution for com- } \\
\text { mercial purposes requires written permission. }\end{array}$
\end{tabular}

Moustapha Ahmed Ramadan

Infection Control Directorate, Ministry of Health

PO Box 12414

Alshamiya (Kuwait)

E-Mail ficas_alex@yahoo.fr 


\section{Introduction}

Hemodialysis is a way of cleansing the blood of toxins, extra salts, and fluids, using a dialysis machine which is generally referred to as an "artificial kidney" [1]. It also helps to maintain the chemical balance of elements such as potassium, sodium and chloride and keeps the blood pressure under control. It is one of the primary means of treating patients with end-stage kidney disease. In 2011, $>395,000$ patients were treated with maintenance hemodialysis in the USA [2]. In Kuwait, 14,892 patients were treated with maintenance hemodialysis in 6 outpatient dialysis centers in 2014, and the number is increasing yearly [3].

Hemodialysis patients are predisposed to developing infections as they are usually immunocompromised due to the development of uremia and other comorbid conditions [4]. Several studies have shown that patients who received treatment in outpatient dialysis facilities were at a high risk of developing infections, probably due to multiple factors such as the need for repeated vascular access through central venous catheters, arteriovenous grafts, or fistulas, and also frequent hospital visits and frequent receipt of antimicrobial drugs [5-7].

Bloodstream infections (BSIs) and localized infections of the vascular access site are a major cause of hospitalization, and are among the leading causes of death in patients undergoing hemodialysis [2]. Measuring and tracking rates of hemodialysis events and utilizing this information are an important part of infection prevention and control measures. Embedded dialysis surveillance, i.e., where the surveillance of dialysis events is embedded in the daily clinical practice, has been shown to be associated with reductions in BSIs, health care burden, patient costs, and the use of antimicrobials [8]. The objective of this study was to determine the difference in dialysis event rates stratified by the type of vascular access and to describe the microbiological profile of the organisms cultured from the blood.

\section{Subjects and Methods}

The study was an epidemiological analysis of dialysis event data collected from March 2013 to February 2016 at the Al Khozam Dialysis Unit, an adult outpatient dialysis center at the Al-Adan Hospital, Kuwait. The dialysis unit has 68 beds: 60 for hemodialysis and 8 for peritoneal dialysis.

The study population comprised patients who required chronic maintenance hemodialysis at the facility. Exclusion criteria were acute hemodialysis and peritoneal dialysis. Dialysis event data were collected based on guidelines outlined in the 2013 Kuwait
National Healthcare-Associated Infections Surveillance System (KNHSS) dialysis event protocol [3].

Three types of dialysis events were reported during the patients' visits to the outpatient dialysis unit: (1) "IV antimicrobials start" where medical records were reviewed to determine if intravenous (IV) antibiotics or antifungals had been prescribed; (2) "positive blood culture" where microbiology reports were reviewed to determine if any bacteria or fungi had been cultured from blood samples collected during an outpatient dialysis unit visit; and (3) "pus, redness or increased swelling at the vascular access site" where medical records were reviewed to assess whether patients had any signs of inflammation at this site. For each event, a second dialysis event of the same type would only be reported for an individual patient if $\geq 21$ days had passed between the 2 episodes. During the 36 -month study period, the medical records of 10,751 patients were reviewed and 1,150 dialysis events were recorded.

\section{Statistical Analysis}

The medical records were reviewed to collect data regarding the incidence of IV antimicrobial use, positive blood culture, and vascular access site inflammation. These data were analyzed using SPSS v18, (IBM, Chicago, IL, USA). Rates were calculated by dividing the number of events by the number of patient-months and multiplying by 100 . Dialysis event rates were stratified by vascular access type (i.e., fistula, graft, and tunneled or nontunneled central line) and expressed per 100 patient-months. The Student $t$ test (2-tailed) was used to check for statistical significance between the different vascular access types (see above). The rates of access-related BSIs, local access infection, and vascular access infection were also calculated as described below.

Access-related BSIs were calculated by determining the number of positive blood culture events where the suspected source of the infection was either vascular or uncertain. Local access infections were calculated by counting the number of pus, redness or increased swelling at the vascular access site events that occurred without a positive blood culture event. Vascular access infections were the sum of the total number of access-related BSIs and the total number of local access infections. Microorganism isolates were thereby classified by vascular access type, and the percentage of multidrug-resistant organisms (MDROs) was calculated from the total isolated number of the same microorganisms over the 3-year study period.

The Kuwait Standing Committee for the Coordination of Health and Medical Research, Ministry of Public Health, approved the study protocol.

\section{Results}

Of the 10,751 patients on dialysis, the access types were: fistula $(n=3,406 ; 31.68 \%)$, graft $(n=256 ; 2.39 \%)$, tunneled central line $(n=6,179 ; 57.47 \%)$, and nontunneled central line $(n=910 ; 8.49 \%)$. Hence, during this period, the most commonly used access type was tunneled central line and the least-used was graft.

The distribution of the dialysis events reported is presented in Table 1. IV antimicrobials start was the most 
Table 1. Distribution of reports according to event details in patients seen at the Al-Khozam Dialysis Unit from March 2013 to February 2016

\begin{tabular}{lr}
\hline & $n(\%)$ \\
\hline I event per report & \\
IV antimicrobials start & $854(82.67)$ \\
Positive blood culture & $19(1.84)$ \\
Pus, redness or swelling & $47(4.55)$ \\
\hline 2 events per report & $60(5.81)$ \\
IV antimicrobials start + positive blood culture & $48(4.65)$ \\
IV antimicrobials start + pus, redness or swelling & $1(0.09)$ \\
Positive blood culture + pus, redness or swelling & \\
\hline 3 events per report & $4(0.39)$ \\
IV antimicrobials start + positive blood culture + pus, & \\
$\quad$ redness or swelling & \\
\hline \multicolumn{2}{l}{ IV, intravenous. }
\end{tabular}

reported event ( $n=854 ; 82.67 \%)$. The 3 events were reported together only 4 times during this study. The IV antimicrobials start event rate was $8.99 / 100$ patientmonths; 167 patients used vancomycin, mostly at a rate of 1.55/100 patient-months. The positive blood culture event rate was $0.78 / 100$ patient-months. Other related rates, such as access-related BSIs, local access infections, and vascular access infections, ranged from 0.75 to 1.64/100 patient-months (Table 2).

The different dialysis events stratified by the type of vascular access are shown in Table 3. The IV antimicrobials start event rate was highest in patients with tunneled central lines (10.29/100 patient-months) and lowest in patients with grafts (7.03/100 patient-months). The rate of the positive blood culture and pus, redness or increased swelling events was highest in patients with nontunneled central lines (1.65/100 and 1.54/100 patient-months, respectively). Differences in IV antimicrobials start, positive blood culture, and pus, redness or increased swelling rates were shown to be significantly higher among patients with tunneled central lines than in those with fistula $(p<0.000)$. The rate of the IV antimicrobials start event in patients with tunneled central lines was statistically significantly higher than in patients with nontunneled central lines $(p=0.04)$. Patients with nontunneled central lines had a significantly higher rate of positive blood culture and pus, redness or increased swelling than patients with fistula ( $p=0.001$ and $p=0.008$, respectively).

The microbiological profile of blood cultures shows that 9 of the 84 positive isolates $(10.71 \%)$ were from pa-
Table 2. Rate of dialysis events

\begin{tabular}{lrc}
\hline & $n$ & Rate \\
\hline Total of dialysis events & 1,150 & 10.7 \\
IV antimicrobials start & 966 & 8.99 \\
Positive blood culture & 84 & 0.78 \\
Pus, redness or swelling & 100 & 0.93 \\
Vancomycin start & 167 & 1.55 \\
Access-related infections & 81 & 0.75 \\
Local access infections & 95 & 0.88 \\
Vascular access infections & 176 & 1.64 \\
\hline
\end{tabular}

The rate is expressed per 100 patient-months. IV, intravenous.

Table 3. Dialysis event rates in relation to vascular access type in the Al-Khozam Dialysis Unit from March 2013 to February 2016

\begin{tabular}{lrc}
\hline & Total & \\
\cline { 2 - 3 } & $n$ & rate \\
\hline Fistula & & \\
Overall rate & & 7.69 \\
IV antimicrobials start & 246 & 7.22 \\
Positive blood culture & 8 & 0.23 \\
Pus, redness or swelling & 8 & 0.23 \\
Vancomycin use & 13 & 0.38 \\
\hline Graft & & \\
Overall rate & & 7.42 \\
IV antimicrobials start & 18 & 7.03 \\
Positive blood culture & 1 & 0.39 \\
Pus, redness or swelling & 0 & 0 \\
Vancomycin use & 2 & 0.78 \\
\hline Tunneled central line & & \\
Overall rate & & 12.53 \\
IV antimicrobials start & 636 & 10.29 \\
Positive blood culture & 60 & 0.97 \\
Pus, redness or swelling & 78 & 1.26 \\
Vancomycin use & 120 & 1.94 \\
\hline Nontunneled central line & & 1.65 \\
Overall rate & & \\
IV antimicrobials start & 66 & \\
Positive blood culture & 15 & \\
Pus, redness or swelling & 14 & \\
Vancomycin use & 32 & \\
\hline & & \\
\hline
\end{tabular}

The rate is expressed per 100 patient-months. IV, intravenous.

tients with fistula or graft, and 75 (89.29\%) were from patients with central lines (tunneled and nontunneled). Gram-negative rods represented the most common group isolated in patients with central lines (46.67\%); however, 
common skin commensals and gram-negative rods were equally identified in patients with fistula and graft $(44.45 \%)$ (Table 4$)$. Six isolates $(7.14 \%)$ cultured over the 3 years were classified as MDROs; 5 of these were cultured from patients with tunneled central lines and 1 was from a patient with a nontunneled central line (Table 5).

\section{Discussion}

Surveillance of dialysis events can help identify patients at risk or highlight areas that are in need of improvement. Health care providers can prevent many of these events by following basic infection prevention recommendations and tracking rates. Additionally, surveillance allows us to spot trends and direct prevention efforts.

In this study, the overall dialysis event rate was (10.7/100 patient-months). This is higher than rates reported in studies conducted in Spain (8.67/100 patientmonths [9] and Ireland (8.79/100 patient-months) [10],

Table 4. Microorganisms isolated from patients with different types of vascular access

\begin{tabular}{lcl}
\hline Microorganism & $\begin{array}{l}\text { Patients with } \\
\text { central line } \\
\text { access, } n(\%)\end{array}$ & $\begin{array}{l}\text { Patients with } \\
\text { fistula/graft } \\
\text { access, } n(\%)\end{array}$ \\
\hline $\begin{array}{l}\text { Staphylococcus aureus } \\
\text { (Other) gram-positive }\end{array}$ & $\begin{array}{l}(5.33) \\
\text { Gram-negative rods }\end{array}$ & $1(11.1)$ \\
Common skin commensals & $29(46.67)$ & - \\
Fungi & $2(2.67)$ & $4(44.45)$ \\
\hline Total & $75(100)$ & $9(44.45)$ \\
\hline
\end{tabular}

but lower than a previous study conducted in Kuwait (11.03/100 patient-months) [11]. Our study had an observation period of 3 years compared to 6 months for the Spanish study [9], 7 months for the Irish study [10], and 13 months for the previous Kuwaiti study [11]. This longer period of surveillance may provide a better representation of dialysis events than the previous studies. However, comparisons of dialysis events across studies may be complicated due to variations in the type of vascular access used in different patient populations, the levels of patient compliance, and the implementation of infection control practices. The higher dialysis event rate in this study may be explained by the relatively greater use of catheters (i.e., in $66 \%$ of the study population) than in the other studies. Vascular access via catheter has been consistently shown to increase a patient's risk of developing an infection [12, 13]. In 2004, the Breakthrough Initiative of Centers for Medicare and Medicaid Services in the USA set a goal of using fistulas for vascular access in $>66 \%$ of all patients receiving hemodialysis [14]. In our study, however, only $32 \%$ of patients had a fistula. Therefore, increased efforts must be made to motivate patients to switch from catheters to fistulas.

The appropriate prescription of antimicrobials is essential to limit the emergence and spread of MDROs in the dialysis patient population $[12,13]$. The IV antimicrobials start event quantifies the patient's exposure to antimicrobials regardless of whether it is related to dialysis or not. We found that the overall IV antimicrobials start event rate was (8.99/100 patient-months); this is higher than is reported elsewhere in the literature [9-11, 15]. When stratifying this event by the type of vascular access, it was highest among patients with tunneled central lines and lowest among patients with grafts. This difference was statistically significant $(p=0.04)$. The high

Table 5. Multidrug-resistant organism isolates identified from dialysis patients

\begin{tabular}{lllll}
\hline Microorganism & Group & Vascular access type & $\begin{array}{l}\text { Isolates, } \\
n\end{array}$ & $\begin{array}{l}\text { MDR isolates, } \\
\left.(\%)^{\mathrm{a}}\right)\end{array}$ \\
\hline Enterobacter cloacae & ESBL & tunneled central line & 5 & $2(40)$ \\
Escherichia coli & ESBL & tunneled central line & 2 & $1(50)$ \\
Klebsiella pneumoniae & ESBL & tunneled central line & 5 & $1(20)$ \\
Staphylococcus aureus & MRSA & nontunneled central line & 5 & $1(20)$ \\
Pseudomonas aeruginosa & MDR-Pseud & tunneled central line & 6 & $1(11.11)$ \\
\hline
\end{tabular}

ESBL, extended spectrum $\beta$-lactamase; MDR, multidrug-resistant; MRSA, methicillin-resistant S. aureus; MDR-Pseud, multidrugresistant Pseudomonas.

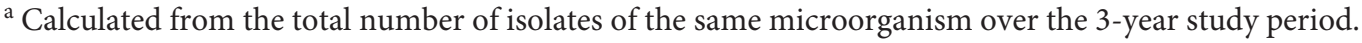


rate of this event indicates that more efforts are needed to prevent the indiscriminate use of antibiotics. The development and use of a standardized antibiotic policy and an antimicrobial stewardship program will help achieve this aim.

This study revealed that the overall positive blood culture event rate was $(0.78 / 100$ patient-months). The rate of positive blood culture in patients undergoing hemodialysis can vary depending upon the type of vascular access. In this study, the positive blood culture rate was highest among patients with temporary nontunneled central lines (1.65/100 patient-months), followed by patients with tunneled central lines $(0.97 / 100$ patientmonths), patients with grafts (0.39/100 patient-months), and then lowest among patients with fistulas $(0.23 / 100$ patient-months). The difference in this rate between nontunneled catheter and fistula use was statistically significant $(p=0.001)$. This distribution is similar to what has been previously reported in the literature [9-11]. It further supports the recommendation that fistula and graft use should be promoted among dialysis patients $[12,13]$. For those patients unable or unwilling to use a fistula or graft, multiple studies have shown that chlorhexidine use at the catheter vascular access site reduces the incidence of BSIs $[12,13,16]$. Appropriate use of this antiseptic may also help reduce BSIs in catheterized patients.

The pus, redness or increased swelling at the vascular access site event (regardless of positive blood culture or IV antimicrobials start) is a new component in dialysis event surveillance launched by the KNHSS in 2013 [3]. No relevant publications are available at this time to compare our reported overall rate (0.93/100 patient-months). Most of the available published data studied pus, redness or swelling in relation to positive blood culture and not as an event on its own. In this study, pus, redness or increased swelling at the vascular access site event was highest among patients with nontunneled central lines and lowest among patients with fistulas, and this difference was shown to be statistically significant $(p=0.008)$. These findings are consistent with reports of higher rates of dialysis events in patients with catheters than in those with fistulas or grafts $[9-11,15]$. This further supports the evidence that fistulas and grafts are safer than vascular catheters.

This study revealed that gram-negative rods were the most commonly isolated microbial group, consistent with the results reported by Sundaresan et al. [17]. With regard to the resistance profiles, we found that $33.3 \%$ of the Enterobacteriaceae isolated were multidrug-resistant (MDR) and that $20 \%$ of the Staphylococcus aureus iso- lated was methicillin-resistant (MRSA). The prevalence of MDR Enterobacteriaceae found in this study is similar to the $36.4 \%$ resistance rate reported by Fram et al. [18]. On reviewing the literature, the resistance of $S$. aureus varied from 2.1 to $38.5 \%[14,18]$. Nevertheless, MRSA has been reported in hemodialysis outpatient facilities, and every effort must be made to reduce the spread of MDROs as they are associated with higher rates of morbidity and mortality [19]. It must be noted that the characteristics of the study population, the hospital infrastructure, and the varying implementation of infection control policies may lead to variations in the microorganisms that are isolated in different facilities.

\section{Conclusion}

Dialysis event rates among patients with fistula or graft were lower than in patients with central line access. IV antimicrobial rate among patients with tunneled central lines was statistically significantly higher than in patients with other types of vascular access. Gram-negative rods were the most commonly isolated microbial group. These findings could facilitate improvements in the planning and coordination of the clinical management of hemodialysis patients, particularly in the areas of antimicrobial stewardship and optimization of vascular access.

References

1 National Institute of Diabetes and Digestive and Kidney Diseases: Choosing a Treatment for Kidney Failure. https://www.niddk.nih. gov/health-information/kidney-disease/kidney-failure/choosing-treatment (last accessed March 16, 2017).

2 US Renal Data System Annual Data Report: Atlas of Chronic Kidney Disease and EndStage Renal Disease in the United States. Bethesda, National Institutes of Health, National Institute of Diabetes and Digestive and Kidney Diseases, 2013. https://www.usrds. org/atlas13.aspx (last accessed March 16, 2017).

3 Kuwait National Healthcare-Associated Infections Surveillance System: Dialysis Event Surveillance. Kuwait, Infection Control Directorate, 2013. http://www.icdkwt.com/surveillence_outcome.php (last accessed March 16, 2017)

4 Ryan SV, Calligaro KD, Dougherty MJ: Management of hemodialysis access infection. Semin Vasc Surg 2004;17:40-44. 
5 Centers for Disease Control and Prevention: Recommendations for preventing transmission of infections among chronic hemodialysis patients. MMWR Morb Mortal Wkly Rep 2001;50:1-46.

6 Centers for Disease Control and Prevention: National Healthcare Safety Network: Dialysis Event Protocol. http://www.cdc.gov/nhsn/ PDFs/pscManual/8pscDialysisEventcurrent. pdf (last accessed March 16, 2017).

7 Stolic R: Most important chronic complications of arteriovenous fistulas for hemodialysis. Med Princ Pract 2013;22:220-228.

8 George A, Tokras JI, Clutterbuck EJ, et al: Reducing dialysis associated-bacteraemia, and recommendations for surveillance in the United Kingdom: a prospective study. BMJ 2006;332:1435

9 Quori A, Baamonde-Laborda E, Garcia-Canton $\mathrm{C}$, et al: Surveillance for infections and other adverse events in dialysis patients in southern Gran Canaria. Nefrologia 2011;31: 457-463.
10 Bajwa R, Casey A, Collier C, et al: Can the NHSN dialysis event protocol be implemented in an Irish dialysis unit? Int J Infect Control 2012;8:1-6.

11 Hussain N, Salama MF, Aly NYA, et al: Infection-related processes during haemodialysis: a study in a general hospital haemodialysis unit. RIF 2010;1:1-6.

12 Association for Professionals in Infection Control and Epidemiology: An APIC Guide: Guide to the Elimination of Infections in $\mathrm{He}$ modialysis 2010. https://apic.org/Professional-Practice/Implementation-guides (last accessed March 16, 2017).

13 Centers for Diseases Prevention and Control: Guidelines for the prevention of intravascular catheter-related infections. https://www.cdc. gov/hai/pdfs/bsi-guidelines-2011.pdf (last accessed March 16, 2017).

14 National Kidney Foundation. KDOQI clinical practice guidelines and clinical practice recommendations for vascular access 2006. Am J Kid Dis 2006;48(suppl 1):s176-s322.
15 Badawy DA, Mowafi HS, Al-Moussa HH Surveillance of dialysis events: 12 -month experience at five outpatient adult haemodialysis centers in Kuwait. J Infect Public Health 2014;7:386-391.

16 Lorente L: What is new for the prevention of catheter-related bloodstream infections? Ann Transl Med 2016;4:119.

17 Sundaresan S, Vincent S, Sivasubramanian S, et al: A study on bacteriological profile of blood culture sample from dialysis patients. $S$ Ind J Biol Sci 2015;1:151-155.

18 Fram D, Okuno MFP, Taminato M, et al: Risk factors for bloodstream infections in patients at a Brazilian haemodialysis center: a case control study. BMC Infect Dis 2015;15:158167.

19 Lee SC, Chen KS, Tsai CJ, et al: An outbreak of methicillin-resistant Staphylococcus aureus infections related to central venous catheters for hemodialysis. Infect Control Hosp Epidemiol 2004;25:678-684. 